\title{
Lactulose ingestion causes an increase in the abundance of gut-resident bifidobacteria in Japanese women: a randomised, double-blind, placebo-controlled crossover trial
}

\author{
Y. Sakai ${ }^{1 *}$, H. Hamano ${ }^{2}$, H. Ochi ${ }^{1}$, F. Abe ${ }^{1}$, K. Masuda ${ }^{3}$ and H. Iino ${ }^{4}$ \\ ${ }^{1}$ Food Ingredients E Technology Institute, RED Division, Morinaga Milk Industry Co., Ltd., 5-1-83 Higashihara, Zama, \\ Kanagawa 252-8583, Japan; ${ }^{2}$ EED Management Department, Morinaga Milk Industry Co., Ltd., 5-1-83 Higashihara, \\ Zama, Kanagawa 252-8583, Japan; ${ }^{3}$ Department of Food Science and Nutrition, Faculty of Life and Environmental Sciences, \\ Showa Women's University, 1-7 Taishidou, Setagaya-ku, Tokyo 154-8533, Japan; ${ }^{4}$ Life Sciences for Living Systems, Graduate \\ School, Showa Women's University, 1-7 Taishidou, Setagaya-ku, Tokyo 154-8533, Japan; yo-sakai@morinagamilk.co.jp
}

Received: 28 May 2020 / Accepted: 17 September 2020

(C) 2020 Wageningen Academic Publishers

\section{OPEN ACCESS @(i) (2) RESEARCH ARTICLE}

\begin{abstract}
The genus Bifidobacterium comprises various bacterial species, and the complement of species within the human intestinal tract differs from individual to individual. The balance of these bifidobacterial species remains poorly understood, although it is known that the abundance of bifidobacteria increases following the ingestion of prebiotics. We previously conducted a randomised, placebo-controlled, double-blind, crossover study of $2 \mathrm{~g} /$ day lactulose ingestion for 2 weeks in 60 Japanese women. To study the effect of lactulose ingestion on each bifidobacterial species, here, we measured the abundance of each of the principal bifidobacterial species. After lactulose ingestion, the log cell counts of the Bifidobacterium adolescentis group ( $8.97 \pm 0.08$ vs $9.39 \pm 0.08, P=0.0019)$, Bifidobacterium catenulatum group $(9.45 \pm 0.10$ vs $9.65 \pm 0.10, P=0.0032)$ and Bifidobacterium longum group $(9.01 \pm 0.07$ vs $9.29 \pm 0.07$, $P=0.0012)$ were significantly higher than in the placebo ingestion control group. However, the log cell counts were similar for Bifidobacterium breve ( $8.12 \pm 0.12$ vs $8.33 \pm 0.12$, $P=0.20$ ), Bifidobacterium bifidum $(9.08 \pm 0.12$ vs $9.42 \pm 0.14$, $P=0.095$ ) and Bifidobacterium animalis subspecies lactis ( $8.65 \pm 0.53$ vs $8.46 \pm 0.46, P=0.77$ ). Cluster analysis of the log cell count data at the bifidobacterial species level revealed three distinct clusters, but the combinations and ratios of the constituent bifidobacteria were not affected by lactulose ingestion. Furthermore, principal coordinate analysis of the intestinal microbiota in the lactulose and placebo ingestion groups using Illumina MiSeq showed no significant differences in the intestinal microbiota as a whole. These results suggest that $2 \mathrm{~g} /$ day lactulose ingestion for 2 weeks significantly increases the abundance of intestinal bifidobacteria, but does not affect the intestinal microbiota as a whole.
\end{abstract}

Keywords: indigestible oligosaccharide, intestinal microbiome, intestinal microbiota, diversity

\section{Introduction}

Bifidobacterium is one of the most well-recognised, beneficial genera of bacteria that inhabits the human intestinal tract (Yaeshima, 1996). Bifidobacteria are present in both the intestines and faeces of both humans and animals. The genus Bifidobacterium comprises 70 species and 10 subspecies (LPSN, http://www.bacterio.net). The species that live in the human gut are designated as human-residential bifidobacteria (HRB) (Wong et al., 2018), and those of animal origin are designated as nonHRB, of which Bifidobacterium animalis is often used as a probiotic, and is consequently detected in human faeces (Kato et al., 2017). These species release vitamins and shortchain fatty acids (SCFAs), such as acetic acid, which are absorbed by the host and metabolised to provide energy. Bifidobacteria are not detected immediately after birth, but after the ingestion of breast milk and/or bottled milk they 
rapidly increase in number and become one of the most abundant types of bacteria, because of the prebiotics and oligosaccharides that are present in human milk and/or the oligosaccharides in bottled milk. However, the abundances of other intestinal bacteria increase after ablactation and the number of bifidobacteria decreases with aging (Odamaki et al., 2016). The abundance of bifidobacteria in Japanese adults is known to be higher than that in the inhabitants of other countries (Nishijima et al., 2016), and these bacteria are thought to be important for the intestinal health of the Japanese population.

Bifidobacteria exist in a dynamic state, and both the abundance and proportions of constituent species change with aging. Bifidobacterial species, such as Bifidobacterium longum subspecies (ssp.) infantis and/or Bifidobacterium bifidum are present in the faeces during infancy, but disappear with aging, whereas species such as Bifidobacterium catenulatum and/or Bifidobacterium adolescentis are present in the faeces during adulthood. Bifidobacterium longum ssp. longum, which is closely related to $B$. longum ssp. infantis, is commonly present in the human gut from infancy to old age (Kato et al., 2017).

Various types of bifidobacteria coexist within humans throughout their life, and the types of bifidobacteria present vary between individuals; however, these changes have not been investigated in detail. For example, it remains to be determined how the proportions of commensal bifidobacterial species change following prebiotic treatment, and whether all, or specific species of bifidobacteria, predominate following prebiotic treatment. If these deficiencies can be rectified, new insight into the usefulness of prebiotics may be provided.

Previously, we conducted a clinical trial to investigate how the defaecation frequency and the number of bifidobacteria in the faeces changed during the ingestion of $2 \mathrm{~g} /$ day lactulose for 2 weeks (Sakai et al., 2019a). Defaecation frequency was found to be significantly higher during lactulose ingestion than during placebo treatment, and the number of bacteria belonging to the genus Bifidobacterium in faeces was significantly higher. In the present study, we analysed the samples obtained in the clinical trial to determine how lactulose intake affects bifidobacterial species, with a specific focus on its effects on the numbers of each bifidobacterial species and the composition ratios in the intestinal microbiota.

\section{Materials and methods}

\section{Trial design}

This study was designed as a randomised, double-blind, placebo-controlled crossover trial, consisting of a preobservation period, then two ingestion periods, which were separated by a washout period. The prebiotic effect of the ingestion of $2 \mathrm{~g} /$ day lactulose for 2 weeks was evaluated (Figure 1). The study was conducted at Showa Women's University (Japan) between May and December 2017, in accordance with the principles of the Declaration of Helsinki. The study protocol was approved by the Institutional Review Board of Showa Women's University (Approval No. 17-10, 7 June 2017). All participants provided their written informed consent. The protocol for this study is registered with the University Hospital Medical Information Network Clinical Trials Registry (No. UMIN000027305).

\section{Participants}

The participants were healthy students, staff members, and affiliates of Showa Women's University, and their families. The inclusion criteria were: (1) age 18-65 years and (2) defaecation frequency $2-4$ times/week. Those over 65 years who may have been affected by other diseases and children under 18 were excluded. In addition, because the primary outcome of the original clinical trial was to investigate the effect of $2 \mathrm{~g} /$ day of lactulose ingestion on defecation frequency, the participants of the study were those who had a defaecation frequency of around once every two days, which is generally considered to represent a tendency toward constipation.

The exclusion criteria were: (1) a severe hepatic, renal, cardiac, gastrointestinal, cerebrovascular, endocrine, metabolic or infectious disease; (2) a history of gastrointestinal resection; (3) gastrointestinal dysfunction, such as irritable bowel syndrome or inflammatory bowel disease; (4) the use of medicines or supplements that could influence defaecation frequency (e.g. antibiotics, probiotics, prebiotics, laxatives, anti-diarrhoeal drugs and fibre); (5) milk allergy; (6) lactose intolerance; (7) participation in another study; and (8) individuals who were judged inappropriate for the study by the investigator or a physician (e.g. who could not meet the deadline for submitting their survey diary or faecal sample, or who could not be contacted during the study period).

\section{Randomisation}

Participants were randomly assigned to group A (lactulose first) or group B (placebo first) using the sealed envelope method. An assignment manager, who was independent of the trial staff, created the allocation order using the replacement block method (block size 4). The allocation ratio was 1:1. According to the allocation order, the test food number was displayed on the test food package for each participant. The correspondence chart between the test food number and the assignment group was hidden from the authors, participants and trial staff, including the intestinal microbiome analyst and the statistician, until the 


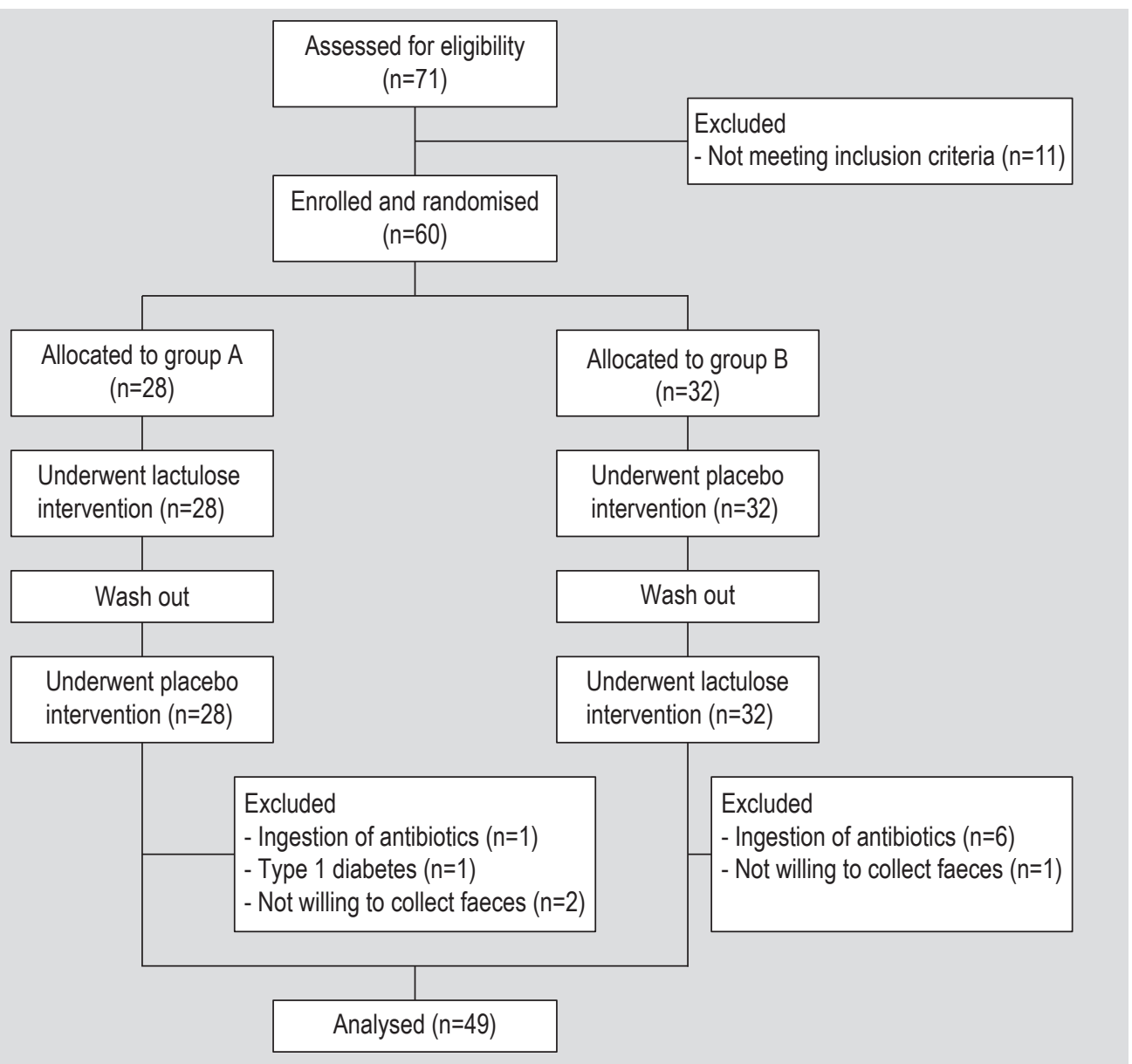

Figure 1. Flow chart of the participant numbers throughout the trial.

completion of the trial. The trial staff assigned the test foods in ascending order of test food number, which corresponded to the order of participant registration.

\section{Intervention}

$2 \mathrm{~g}$ portions of lactulose crystal anhydrate powder (MLC-97, $\geq 97 \%$, Morinaga Milk Industry Co., Ltd., Tokyo, Japan) were provided in aluminium sachets. Glucose crystal anhydrate powder (Nihon Shokuhin Kako Co., Ltd., Tokyo, Japan) was used as the placebo, because this could not be visually discriminated from the lactulose. Participants in group A received lactulose during the first intervention period and the placebo during the second intervention period, for 2 weeks each, whereas participants in group $B$ received the placebo during the first intervention period and lactulose during the second intervention period. A 3-week washout period was included between the two intervention periods (Figure 2). The time of day for ingestion was not specified. The participants were instructed in advance to avoid the use of pharmaceuticals and supplements (e.g. antibiotics, laxatives, anti-diarrhoeal drugs, probiotics, prebiotics and fibre), which could affect defaecation during the study period.

\section{DNA extraction}

Faecal samples were collected before and at the end of each intervention period (Figure 2), and were immediately placed in storage at $\leq 18^{\circ} \mathrm{C}$ until they arrived at the laboratory, and

\begin{tabular}{|l|l|c|c|c|}
\hline Selection period & $\begin{array}{c}\text { Pre-observation period } \\
2 \text { weeks }\end{array}$ & $\begin{array}{c}\text { 1st intervention period } \\
2 \text { weeks }\end{array}$ & $\begin{array}{c}\text { Washout period } \\
3 \text { weeks }\end{array}$ & $\begin{array}{c}\text { 2nd intervention period } \\
2 \text { weeks }\end{array}$ \\
\hline
\end{tabular}

Figure 2. Trial design. Faecal sample collections are indicated by arrows. 
at $-80{ }^{\circ} \mathrm{C}$ thereafter. Because the participants' defaecation frequencies were 2-4 times per week and it was difficult to designate a specific date for sample collection, this was scheduled to occur before administration and on the last day of administration \pm 2 days. DNA was extracted from these samples, as described by Sugahara et al. (2015), and used to determine both the log cell count of Bifidobacterium species and their percentages within the faecal microbiome.

\section{Log cell count of bifidobacterial}

To determine the log cell count of bifidobacterial species per gram of faeces, a quantitative PCR method was used. The specific primers used in the PCR are shown in Supplementary Table S1. For the genus Bifidobacterium, a standard curve was prepared using dilutions of B. longum JCM $1217^{\mathrm{T}}\left(\mathrm{ATCC} 15707^{\mathrm{T}}\right)$ cells. For the $B$. adolescentis group (comprising B. adolescentis genotypes $A$ and B), a standard curve was prepared using dilutions of B. adolescentis JCM1275 ${ }^{\mathrm{T}}$ cells. For B. animalis ssp. lactis, a standard curve was prepared using dilutions of B. animalis ssp. lactis JCM $1190^{\mathrm{T}}$ cells. For B. bifidum, a standard curve was prepared using dilutions of $B$. bifidum JCM $1255^{\mathrm{T}}$ cells. For B. breve, a standard curve was prepared using dilutions of $B$. breve JCM $1192^{\mathrm{T}}$ cells. For the B. catenulatum group (B. catenulatum and Bifidobacterium pseudocatenulatum), a standard curve was prepared using dilutions of B. pseudocatenulatum JCM1200 ${ }^{\mathrm{T}}$ cells. For the B. longum group (B. longum ssp. infantis, B. longum ssp. longum and Bifidobacterium longum ssp. suis), a standard curve was prepared using dilutions of B. longum $\mathrm{JCM} 217^{\mathrm{T}}\left(\mathrm{ATCC} 15707^{\mathrm{T}}\right)$ cells. For B. longum ssp. longum, a standard curve was prepared using dilutions of B. longum JCM1217 ${ }^{\mathrm{T}}\left(\mathrm{ATCC} 15707^{\mathrm{T}}\right)$ cells. The data before and at the end of intervention were logarithmically transformed and statistically analysed.

\section{Percentages of bifidobacterial species in the faecal microbiome}

To determine the percentage of each bacterial taxon of interest in the faecal microbiome, the same DNA extract that was used to determine the log cell counts of Bifidobacterium was sequenced using Illumina MiSeq (Illumina, Inc., San Diego, CA, USA). Amplification of $16 \mathrm{~S}$ rRNA by PCR and sequencing using Illumina MiSeq were performed as previously reported by Kato et al. (2018). The V3-V4 regions of the bacterial $16 \mathrm{~S}$ rRNA genes were then amplified by PCR using a TaKaRa Ex Taq HS Kit (TaKaRa Bio, Shiga, Japan) and the primer set Tru357F (5'-CGCTCTTCCGATCTCTGTA CGGRAGGCAGCAG-3') and Tru806R (5'-CGCTCTTCC GATCTGACGGACTACHVGGGTWTCTAAT-3'). Reads containing the bacteriophage phiX DNA sequence in the Genome Reference Consortium human build 38 (GRCh38) were identified by mapping them against Bowtie2 (version
2.2.6) and discarded. Then, the sequences were analysed using the QIIME2 software package, version 2017.10 (https://qiime2.org/). Potential chimeric sequences were removed using DADA2 (Callahan et al., 2016), then 30 and 90 bases of the 3 ' region of the forward and reverse reads were trimmed, respectively. Taxonomic classification was performed in QIIME2 software using a Naive Bayes classifier trained on Greengenes 13.8, with a 99\% threshold for operational taxonomic unit (OTU) full-length sequences. UniFrac distances were calculated using QIIME2 software. The data collected at the end of the intervention period were statistically analysed.

\section{Detection rate}

The detection rate was defined as the number of people in whom bifidobacteria were detected using quantitative PCR divided by the total number of participants who submitted faecal samples, for each bifidobacterial species. The detection rates were determined both prior to (the leftmost arrow in Figure 2) and following the intake of lactulose or placebo. The detection of bifidobacteria was evaluated on the basis of the detection limits of the quantitative PCR: a log cell count/g faeces of 6 .

\section{Statistical methods}

On the basis of the results of a preliminary trial (Sakai et al., 2019b), the number of participants required was calculated using a two-tailed significance level of $5 \%$ and a power of $90 \%$, with the assumption that the difference in defaecation frequency between the groups would be 0.7 and the standard deviation would be 1.5. The number of participants required was calculated to be 51 . Assuming that the combined withdrawal and dropout rate would be $\sim 10 \%$, the number of participants required was determined to be 60 .

Statistical analysis was conducted per protocol set (PPS), meaning that the participants who took medicines or supplements that could affect defaecation, or those who had a disease, were excluded from the analysis.

The lactulose and placebo groups were compared using a linear mixed model, in which the test food group and timing were fixed effects, and participant identity was a variable effect. The least mean square value and standard error for each group, the difference between the groups, and the associated 95\% confidence interval (CI) and $P$-value were calculated. The two-tailed significance level was set at $5 \%$.

Samples taken before and after lactulose ingestion, and before and after placebo ingestion, were analysed using a paired $t$-test. The simple mean and standard deviation for each group, and the $P$-value were calculated. The two-tailed significance level was set at $5 \%$. 
The relationship between the overall gut microbiome composition and lactulose ingestion was assessed using unweighted (qualitative) and weighted (quantitative) UniFrac distance matrices and permutational multivariate analysis of variance (PERMANOVA). The number of permutations was set to 10,000 . Principal coordinate analysis (PCoA) plots were generated using the first two principal coordinates.

Statistical analyses of the data were performed using JMP 13.2 (SAS Institute Inc., Cary, NC, USA). To construct the dendrogram, XLSTAT 2014.1.09 (Addinsoft Inc., New York, NY, USA) was used. The unweighted and weighted UniFrac distances obtained from the microbial data using the QIIME2 software package were used for PCoA. PERMANOVA was performed using the package 'vegan' in R software (version 3.6.0).

\section{Results}

\section{Participants}

Sixty healthy participants were enrolled in the study, of whom 28 were assigned to group A and 32 to group B (Figure 1). There were no male participants in the study and no participants dropped out. Seven participants who used antibiotics during the trial and one who had type I diabetes were excluded. Three participants were not willing to collect faeces; therefore, this additional analysis ultimately included 49 participants. There was a risk of bias due to the use of PPS analysis. The mean age the 49 participants in this additional analysis was $20.3 \pm 2.4$ years (range, 18 - 31 years).

\section{Detection rate}

The detection rate of each bifidobacterial species before the test food intervention is shown in Table 1. The genus Bifidobacterium was present in $100 \%$ of the participants in this trial, of which the $B$. adolescentis group was present in $49.0 \%$, B. animalis ssp. lactis was present in $32.7 \%$, B. bifidum was present in $14.3 \%, B$. breve was present in $53.1 \%$, the $B$. catenulatum group was present in $63.3 \%$, the B. longum group was present in $89.8 \%$, and B. longum ssp. longum was present in $89.8 \%$.

Table 2 shows the detection rate for each bifidobacterial species after placebo or lactulose treatment. The genus Bifidobacterium was present in $100 \%$ of samples in each group. Specifically, the $B$. adolescentis group was present in 49.0 and $44.9 \%$, B. animalis ssp. lactis was present in 20.4 and $28.6 \%$, B. bifidum was present in 16.3 and $16.3 \%$, B. breve was present in 51.0 and $57.1 \%$, the $B$. catenulatum group was present in 63.3 and $61.2 \%$, the $B$. longum group was present in 89.8 and $89.8 \%$, and B. longum ssp. longum was present in 89.8 and $89.8 \%$ of each group, respectively.
Table 1. Detection rate of each bifidobacterial species before the interventions. ${ }^{1}$

\begin{tabular}{|c|c|c|}
\hline \multirow[t]{2}{*}{ Bifidobacterial species } & \multicolumn{2}{|c|}{ Before the test food intervention } \\
\hline & $\begin{array}{l}\text { Number } \\
\text { detected }\end{array}$ & $\begin{array}{l}\text { Detection } \\
\text { rate }(\%)\end{array}$ \\
\hline Genus Bifidobacterium & 49 & 100.0 \\
\hline Bifidobacterium adolescentis group & 24 & 49.0 \\
\hline Bifidobacterium animalis ssp. lactis & 16 & 32.7 \\
\hline Bifidobacterium bifidum & 7 & 14.3 \\
\hline Bifidobacterium breve & 26 & 53.1 \\
\hline Bifidobacterium catenulatum group & 31 & 63.3 \\
\hline Bifidobacterium longum group & 44 & 89.8 \\
\hline Bifidobacterium longum ssp. longum & 44 & 89.8 \\
\hline
\end{tabular}

\section{Log cell counts for Bifidobacterium}

The log cell counts for bifidobacterial species are shown in Table 3 and Supplementary Figure S1. In Table 3, the least square mean and standard error are used, because this result shows the comparison between after lactulose and placebo ingestion in the crossover trial (Choi et al., 2014). After lactulose ingestion, the log cell counts, not only of the genus Bifidobacterium ( $9.16 \pm 0.06$ vs $9.53 \pm 0.06$, $P<0.0001)$, but also of the $B$. adolescentis group $(8.97 \pm 0.08$ vs $9.39 \pm 0.08, P=0.0019)$, the $B$. catenulatum group $(9.45 \pm 0.10$ vs $9.65 \pm 0.10, P=0.0032)$, and the $B$. longum group $(9.01 \pm 0.07$ vs $9.29 \pm 0.07, P=0.0012$ ), including $B$. longum ssp. longum $(9.04 \pm 0.06$ vs $9.29 \pm 0.07, P=0.0008)$ were significantly higher than in the placebo group. The log cell counts were similar for $B$. breve ( $8.12 \pm 0.12$ vs $8.33 \pm 0.12, P=0.20$ ), $B$. bifidum (9.08 \pm 0.12 vs $9.42 \pm 0.14, \mathrm{P}=0.095)$ and $B$. animalis ssp. lactis $(8.65 \pm 0.53$ vs $8.46 \pm 0.46, P=0.77)$ in the two groups.

The abundances of B. animalis ssp. lactis, B. bifidum and $B$. breve, which did not show significant differences between lactulose and placebo ingestion, were also compared before and after the ingestion period, to further characterise the bifidobacterial counts within the groups. The results are shown in Table 4 and Supplementary Figure S1, in which the simple mean values and standard deviations are quoted, because they show comparisons between participants before and after lactulose and placebo ingestion. The log cell count of $B$. breve increased significantly $(7.93 \pm 0.87 \mathrm{vs}$ $8.24 \pm 0.87, P=0.031)$, whereas those of $B$. bifidum $(9.17 \pm 0.42$ vs $9.34 \pm 0.23, P=0.10)$ and $B$. animalis ssp. lactis $(8.14 \pm 1.26$ vs $8.55 \pm 1.40, P=0.44)$ did not. With respect to the other bifidobacterial species, the log cell counts for the genus 
Table 2. Detection rate for each bifidobacterial species after the test food interventions. ${ }^{1}$

\begin{tabular}{|c|c|c|c|c|}
\hline \multirow[t]{2}{*}{ Bifidobacterial species } & \multicolumn{2}{|l|}{ Placebo } & \multicolumn{2}{|l|}{ Lactulose } \\
\hline & Number detected & Detection rate $(\%)$ & Number detected & Detection rate $(\%)$ \\
\hline Genus Bifidobacterium & 49 & 100.0 & 49 & 100.0 \\
\hline Bifidobacterium adolescentis group & 24 & 49.0 & 22 & 44.9 \\
\hline Bifidobacterium animalis ssp. lactis & 10 & 20.4 & 14 & 28.6 \\
\hline Bifidobacterium bifidum & 8 & 16.3 & 8 & 16.3 \\
\hline Bifidobacterium breve & 25 & 51.0 & 28 & 57.1 \\
\hline Bifidobacterium catenulatum group & 31 & 63.3 & 30 & 61.2 \\
\hline Bifidobacterium longum group & 44 & 89.8 & 44 & 89.8 \\
\hline Bifidobacterium longum ssp. longum & 44 & 89.8 & 44 & 89.8 \\
\hline
\end{tabular}

Table 3. Log cell counts for bifidobacterial species after the test food interventions. ${ }^{1}$

\begin{tabular}{|c|c|c|c|c|c|}
\hline Bifidobacterial species & $\mathrm{n}$ & Placebo & Lactulose & Delta $\left(95 \% \mathrm{Cl}^{2}\right)$ & $P$-value \\
\hline Genus Bifidobacterium & 49 & $9.16 \pm 0.06$ & $9.53 \pm 0.06$ & $0.37(0.23$ to 0.49$)$ & $<0.0001$ \\
\hline Bifidobacterium adolescentis group & 22 & $8.97 \pm 0.08$ & $9.39 \pm 0.08$ & $0.42(0.17$ to 0.66$)$ & 0.0019 \\
\hline Bifidobacterium animalis ssp. lactis & 11 & $8.65 \pm 0.53$ & $8.46 \pm 0.46$ & $0.19(-3.27$ to 2.89$)$ & 0.77 \\
\hline Bifidobacterium bifidum & 8 & $9.08 \pm 0.12$ & $9.42 \pm 0.14$ & $0.34(-0.08$ to 0.75$)$ & 0.095 \\
\hline Bifidobacterium breve & 26 & $8.12 \pm 0.12$ & $8.33 \pm 0.12$ & $0.21(-0.11$ to 0.55$)$ & 0.20 \\
\hline Bifidobacterium catenulatum group & 30 & $9.45 \pm 0.10$ & $9.65 \pm 0.10$ & $0.20(0.07$ to 0.33$)$ & 0.0032 \\
\hline Bifidobacterium longum group & 44 & $9.01 \pm 0.07$ & $9.29 \pm 0.07$ & $0.28(0.13$ to 0.43$)$ & 0.0012 \\
\hline Bifidobacterium longum ssp. longum & 44 & $9.04 \pm 0.06$ & $9.29 \pm 0.07$ & $0.25(0.12$ to 0.38$)$ & 0.0008 \\
\hline
\end{tabular}

Bifidobacterium (9.10 \pm 0.81 vs $9.48 \pm 0.65, P<0.0001)$, the $B$. adolescentis group ( $8.82 \pm 0.81$ vs $9.32 \pm 0.54, P=0.00013)$, the $B$. catenulatum group $(9.34 \pm 0.78$ vs $9.64 \pm 0.57, P=0.034)$ and the $B$. longum group $(9.04 \pm 0.55$ vs $9.29 \pm 0.58, P=0.0010)$, including $B$. longum ssp. longum (9.08 \pm 0.53 vs $9.30 \pm 0.53$, $P=0.0017)$, significantly increased in the lactulose group. However, there were no significant changes in any of the bifidobacterial species in the placebo group during the study period.

\section{Percentages of the faecal microbiome}

The percentages of the top 10 most abundant species of the faecal microbiome after placebo ingestion are shown in Table 5, in which the least square mean and standard error are shown. The least square mean values for the percentages of the genus Bifidobacterium were $18.1 \pm 1.4$ for the placebo group and $25.3 \pm 1.4$ for the lactulose group. The difference between the groups was 7.2 (95\% CI 2.9$11.4, P=0.0014)$. Bacteria other than those belonging to the genus Bifidobacterium, which included the genus Blautia, and which predominated after placebo intake, did not significantly change during the study, and the abundance ranking of the other species represented in the faecal microbiome also remained largely unchanged.

Figure 3 shows the results of PCoA of the microbiota after lactulose or placebo ingestion. The differences between the groups were not significant using unweighted UniFrac distances $(P=0.99)$ or weighted UniFrac distances $(P=0.085)$.

\section{Dendrogram}

The dendrogram in Figure 4 shows the results of cluster analysis using the cell counts for bifidobacteria of each species. Three clusters were apparent. For almost all the participants, the clusters remained the same after lactulose or placebo ingestion. Supplementary Table S2 shows the observations that made up each cluster in the dendrogram. All the participants except S032 and S057 belonged to the same cluster both after lactulose ingestion and after placebo ingestion. 
Table 4. Log cell counts of bifidobacterial species before and after the test food interventions. ${ }^{1}$

\begin{tabular}{|c|c|c|c|c|c|c|c|c|}
\hline \multirow[t]{2}{*}{ Bifidobacterial species } & \multirow[t]{2}{*}{$n$} & \multicolumn{2}{|l|}{ Placebo } & \multirow[t]{2}{*}{$P$-value } & \multirow[t]{2}{*}{$n$} & \multicolumn{2}{|l|}{ Lactulose } & \multirow[t]{2}{*}{$P$-value } \\
\hline & & before & after & & & before & after & \\
\hline Genus Bifidobacterium & 49 & $9.24 \pm 0.77$ & $9.21 \pm 0.71$ & 0.60 & 49 & $9.10 \pm 0.81$ & $9.48 \pm 0.65$ & $<0.0001$ \\
\hline Bifidobacterium adolescentis group & 21 & $8.95 \pm 0.81$ & $9.04 \pm 0.57$ & 0.70 & 22 & $8.82 \pm 0.81$ & $9.32 \pm 0.54$ & 0.00013 \\
\hline Bifidobacterium animalis ssp. lactis & 6 & $9.66 \pm 0.96$ & $9.10 \pm 1.00$ & 0.23 & 10 & $8.14 \pm 1.26$ & $8.55 \pm 1.40$ & 0.44 \\
\hline Bifidobacterium bifidum & 8 & $9.28 \pm 0.26$ & $9.13 \pm 0.48$ & 0.30 & 7 & $9.17 \pm 0.42$ & $9.34 \pm 0.23$ & 0.10 \\
\hline Bifidobacterium breve & 23 & $8.19 \pm 0.83$ & $8.23 \pm 0.91$ & 0.72 & 25 & $7.93 \pm 0.87$ & $8.24 \pm 0.87$ & 0.031 \\
\hline Bifidobacterium catenulatum group & 30 & $9.49 \pm 0.64$ & $9.46 \pm 0.78$ & 0.73 & 30 & $9.34 \pm 0.78$ & $9.64 \pm 0.57$ & 0.034 \\
\hline Bifidobacterium longum group & 44 & $9.08 \pm 0.63$ & $9.02 \pm 0.65$ & 0.41 & 43 & $9.04 \pm 0.55$ & $9.29 \pm 0.58$ & 0.0010 \\
\hline Bifidobacterium longum ssp. longum & 44 & $9.10 \pm 0.61$ & $9.04 \pm 0.63$ & 0.43 & 43 & $9.08 \pm 0.53$ & $9.30 \pm 0.53$ & 0.0017 \\
\hline
\end{tabular}

Table 5. Bacterial taxa abundance, expressed as percentages of the faecal microbiome after the test food intervention. ${ }^{1}$

\begin{tabular}{lccccc} 
Genus & $\mathbf{n}$ & Placebo & Lactulose & Delta $\left(95 \% \mathrm{Cl}^{2}\right)$ & $P$-value \\
Blautia & & & & & \\
Bifidobacterium & 49 & $22.8 \pm 1.1$ & $21.7 \pm 1.1$ & $7.1(-2.0$ to 4.2$)$ & 0.47 \\
Lachnospiraceae gen. & 49 & $18.1 \pm 1.4$ & $25.3 \pm 1.4$ & $7.2(2.9$ to 11.4$)$ & 0.0014 \\
Coprococcus & 49 & $7.8 \pm 0.6$ & $6.8 \pm 0.6$ & $1.0(-0.5$ to 2.4$)$ & 0.18 \\
Lachnospiraceae Ruminococcus & 49 & $6.9 \pm 0.5$ & $7.0 \pm 0.5$ & $0.1(-1.2$ to 1.5$)$ & 0.82 \\
Faecalibacterium & 49 & $6.6 \pm 0.5$ & $5.3 \pm 0.5$ & $1.3(-0.1$ to 2.7$)$ & 0.061 \\
Roseburia & 49 & $5.1 \pm 0.6$ & $4.9 \pm 0.6$ & $0.2(-1.8$ to 2.1$)$ & 0.87 \\
Streptococcus & 49 & $4.6 \pm 0.7$ & $3.7 \pm 0.7$ & $0.9(-1.1$ to 3.0$)$ & 0.37 \\
Ruminococcus & 49 & $3.5 \pm 0.7$ & $4.0 \pm 0.7$ & $0.5(-1.3$ to 2.4$)$ & 0.58 \\
Bacteroides & 49 & $3.3 \pm 0.4$ & $3.5 \pm 0.4$ & $0.2(-0.9$ to 1.3$)$ & 0.71 \\
\hline
\end{tabular}

${ }^{1}$ Values are expressed as the least square mean \pm standard error (linear mixed model).

${ }^{2} \mathrm{Cl}=$ confidence interval.
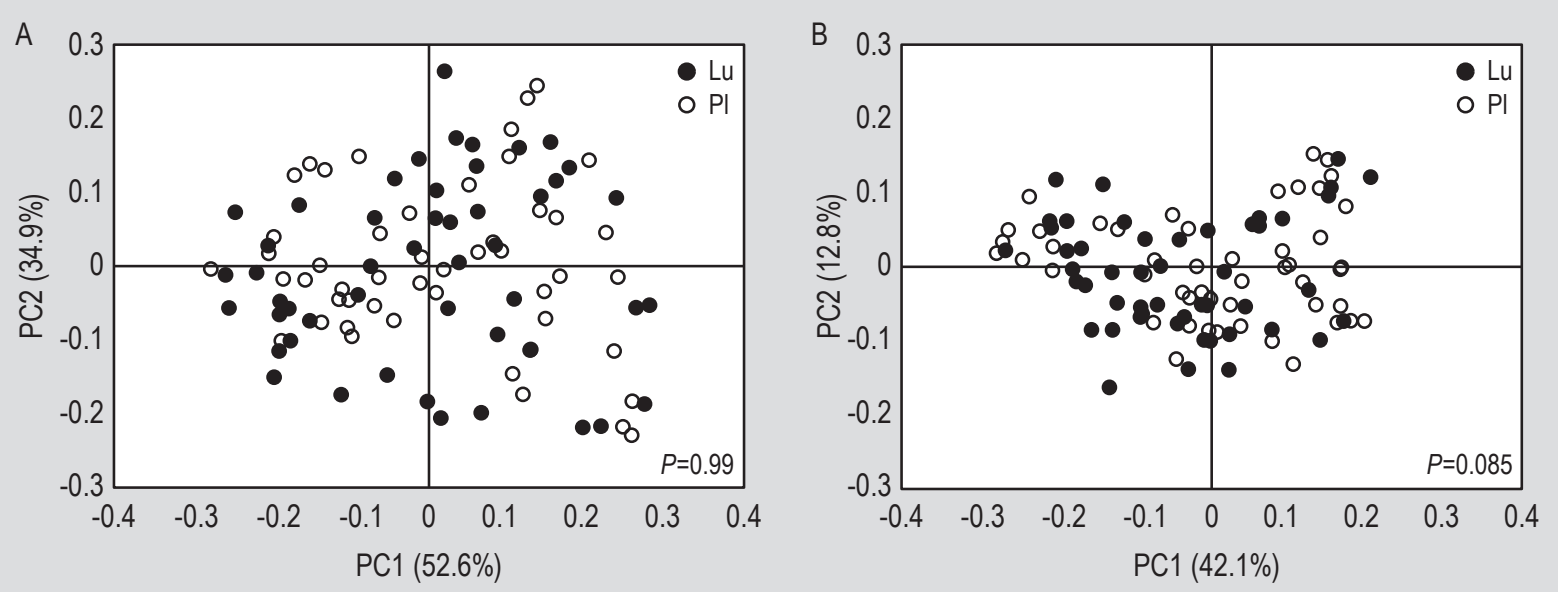

Figure 3. Principal coordinate analysis score plots generated using the first two principal coordinates (PC): PC1 and PC2. Data were analysed using PERMANOVA and were based on (A) unweighted UniFrac distances or (B) weighted UniFrac distances. The differences between groups were not significant using the unweighted UniFrac distances $(P=0.99)$ or weighted UniFrac distances $(P=0.085)$. 


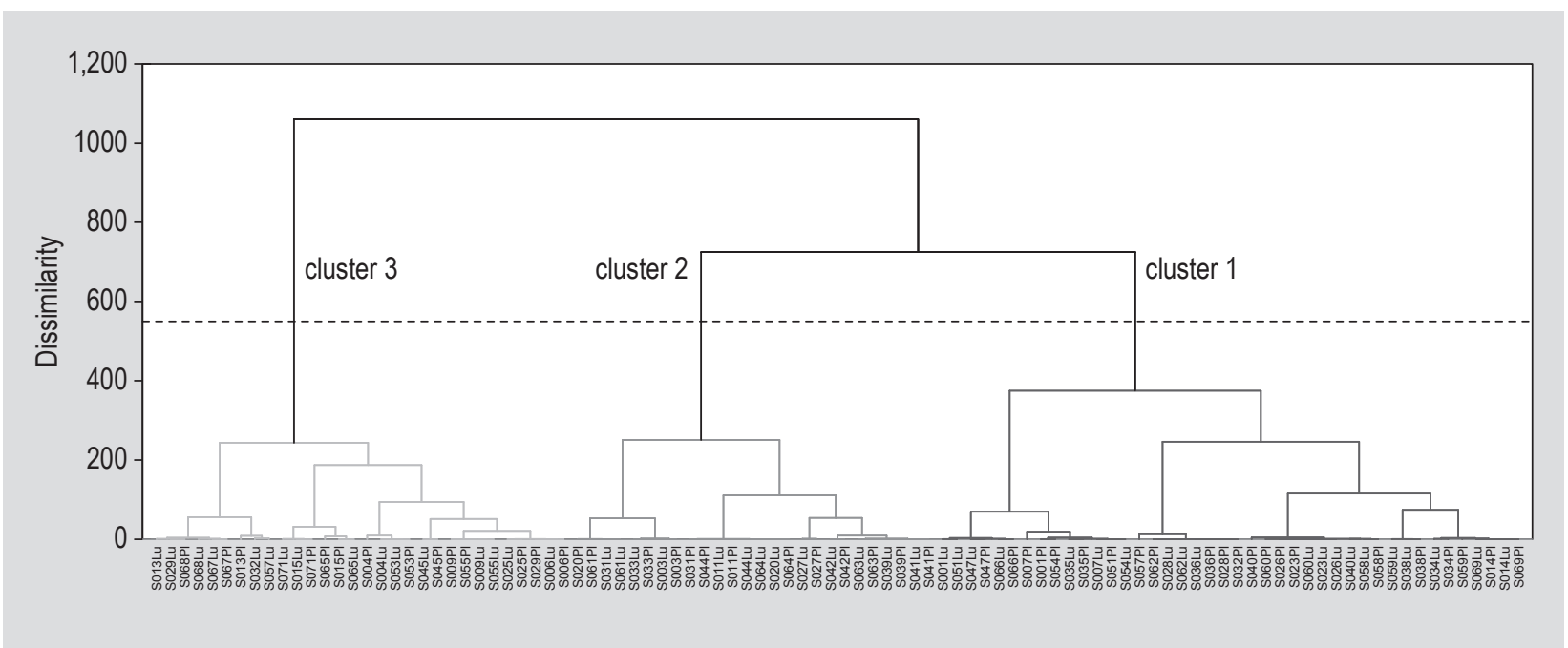

Figure 4. Dendrogram constructed using the logarithmically transformed cell counts for bifidobacterial species.

Supplementary Figure S2A shows the ratios of bifidobacterial species in each cluster separately for lactulose and placebo ingestion, which were calculated using untransformed bifidobacterial cell counts. Supplementary Figures S2B shows the cumulative untransformed cell counts for the bifidobacterial species in each cluster, separately for lactulose and placebo ingestion.

The bifidobacterial biotas of the participants belonging to cluster 1 were similar in that they contained the $B$. adolescentis group, $B$. catenulatum group, $B$. longum group and $B$. bifidum, but $B$. animalis ssp. lactis was uncommon, only being present on one branch of the dendrogram. All the participants possessed the $B$. adolescentis group and B. longum group, and $81 \%$ of the participants possessed the $B$. catenulatum group. Cluster 1 branched on the basis of the presence or absence of $B$. breve. The bifidobacterial biotas of the participants belonging to cluster 2 were similar, in that they possessed the B. catenulatum group and $B$. longum group, but lacked the $B$. adolescentis group. Cluster 2 also branched on the basis of the presence/absence of $B$. breve. In cluster 2, the B. catenulatum group showed a significantly and consistently higher log cell count than the $B$. longum group $(9.98 \pm 0.30$ vs $9.20 \pm 0.40, P<0.0001$ by paired $t$-test, including both the post-lactulose ingestion and post-placebo ingestion data) (Figure 5). Furthermore, the log cell count for the B. catenulatum group in cluster 2 was significantly higher than that in cluster $1(9.98 \pm 0.30$ vs $9.21 \pm 0.57, P<0.0001$, by $t$-test, including both the postlactulose ingestion and post-placebo ingestion data).

Cluster 3 was characterised by the possession of the $B$. longum group and $B$. breve. Almost none of the participants possessed the B. catenulatum group and/or B. bifidum. In cluster 3 , the branches represented the presence or absence of the $B$. adolescentis group, the $B$. longum group and B. breve.

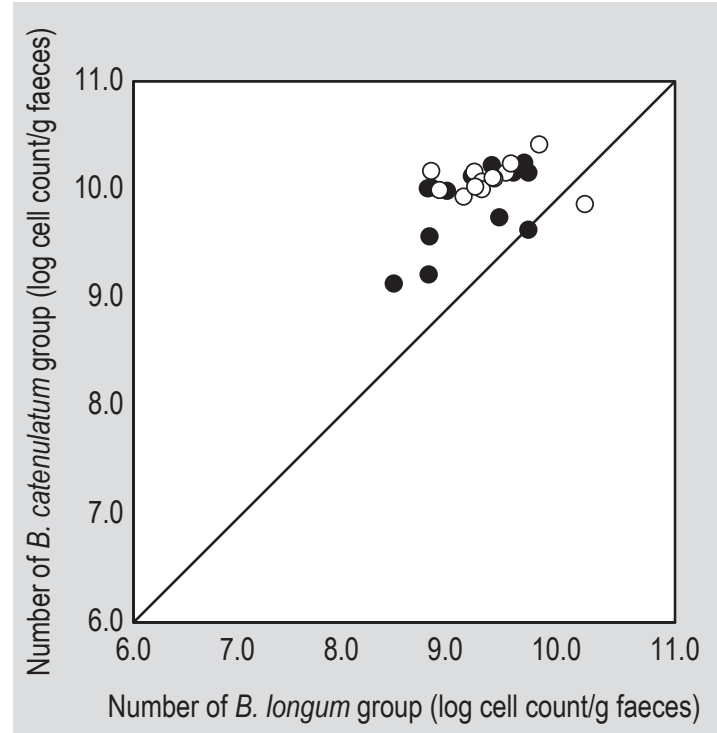

Figure 5. Results of correlation analysis of the log cell counts of the Bifidobacterium catenulatum group and Bifidobacterium longum group in cluster 2. $\bigcirc$ after lactulose ingestion; - after placebo ingestion. The B. catenulatum group showed a consistently higher log cell count than the $B$. longum group $(9.98 \pm 0.30$ vs $9.20 \pm 0.40, P<0.0001$, paired t-test, including both after lactulose ingestion and after placebo ingestion data).

\section{Discussion and conclusions}

This is the first study to determine the effect of oral lactulose on the abundance of each bifidobacterial species. However, one limitation of the study was that all of the participants were young Japanese women, therefore, it may be difficult to extrapolate the results to the whole population or to populations in other geographical regions. 
There have been several studies regarding the relationship between lactulose ingestion and bifidobacteria in faeces. In particular, studies conducted in healthy Japanese people have all shown a significant increase in numbers (Mizota et al., 2002; Sakai et al., 2019a; Tomoda et al., 1991). Tomoda et al. (1991) reported that the number of bifidobacteria was significantly higher in participants that had consumed yogurt containing $0.65 \mathrm{~g}$ of lactulose than in those who had consumed yogurt that did not contain lactulose. Mizota et al. (2002) also reported significantly higher numbers of bifidobacteria when beverages containing $4 \mathrm{~g}$ of lactulose were consumed, compared with the consumption of beverages not containing lactulose. However, in these studies, only the abundance of the genus Bifidobacterium was assessed, and therefore it remained to be determined which bifidobacterial species contributed to the greater abundance of this genus. Similarly, in the studies by Ballongue et al. (1997), Tuohy et al. (2002), Bouhnik et al. (2004a), Bouhnik et al. (2004b) and Vanhoutte et al. (2006), who studied healthy non-Japanese people, the relationships between lactulose ingestion and bifidobacterial abundance in faeces were also assessed, but it also remains unknown which bifidobacterial species contributed to the significant differences in the abundance of the genus Bifidobacterium. In addition, it has become relatively clear that the bifidobacterial species change between generations (Kato et al., 2017). Bifidobacteria do not form a majority of the microbiota in non-Japanese adults (Nishijima et al., 2016), and the identity of the bifidobacterial species in non-Japanese people have not been studied in detail. In the present study, we have shown that the increase in bifidobacteria in the Japanese participants was mainly due to increases in the numbers of the species of bifidobacteria that form the majority of the bacterial population in Japanese people and in each participant, such as the B. longum group, B. catenulatum group and B. adolescentis group.

In previous studies of the bifidobacteria species in the Japanese population under normal conditions, the B. longum group was the most frequently identified species in teenagers and in people in their twenties, followed by the B. adolescentis group and B. catenulatum group (Kato et al., 2017). Similar results were obtained in the present study. In addition to the comparisons at the genus level, the numbers of bacteria belonging to the $B$. adolescentis group, B. catenulatum group and B. longum group were significantly higher after lactulose ingestion than after placebo ingestion, and the log cell counts were also significantly increased by lactulose ingestion. The log cell count for $B$. breve after lactulose ingestion was not significantly higher than after placebo ingestion; however, it was significantly higher than before lactulose ingestion. For two participants, the total log cell counts of bacteria belonging to the genus Bifidobacterium, which were determined using the primers g-Bifid-F and g-Bifid-R, could not be fully explained by the bifidobacterial species that were quantified in the present study. The bifidobacteria possessed by some participants may have been HRB species, such as Bifidobacterium angulatum or Bifidobacterium dentium, which were not analysed in the present study, or non-HRB species, such as Bifidobacterium thermophilum or Bifidobacterium animalis ssp. animalis. Non-HRB might become the predominant bifidobacterial species in the human intestine if it was continuously ingested. However, the participants were prohibited from taking probiotics in the present study period. The lack of quantification of these types of bifidobacteria should be taken into consideration when interpreting the findings of the present study.

The detection rate of $B$. bifidum was low and its abundance did not increase significantly during the trial. Because it is generally considered to be an infant-specific species of Bifidobacterium, its low detection rate in this trial was as expected. However, the log cell count of B. bifidum tended to be higher after lactulose ingestion than after placebo ingestion. The detection rate of $B$. animalis ssp. lactis, which generally resides in animal intestines, was similar to those of previous reports (Kato et al., 2017). For example, the B. longum group was identified in one participant throughout the trial period, whereas B. animalis ssp. lactis was not consistently identified during the study period. This might be due to the fact that participants consumed B. animalis ssp. lactis without realising it, even though they were instructed to refrain from eating foods containing probiotics.

As described above, most of the intestinal bifidobacteria seemed to increase in abundance following lactulose ingestion. According to cluster analysis that used a dendrogram based on the log cell count of each bifidobacterial species, participants were divided into three clusters. Cluster 3 was mainly separated from clusters 1 and 2 by the presence/absence of the B. catenulatum group, whereas clusters 1 and 2 were separated by the presence/ absence of the B. adolescentis group. Only S032 and S057 belonged to different clusters after lactulose ingestion and after placebo ingestion, which indicated a difference in the composition of the bifidobacteria associated with lactulose and placebo ingestion in these individuals. Further inspection revealed that the B. longum group and $B$. adolescentis group were detected after placebo ingestion in both participants, whereas B. breve and B. animalis ssp. lactis were detected after lactulose ingestion, in addition to the B. longum group and B. adolescentis group, in both participants. When lactulose had not been ingested, the $\log$ cell count of $B$. breve was below the detection limit. If $B$. breve had also been detected after placebo ingestion, it would have been presumed that this species belonged to cluster 3, both after lactulose ingestion and after placebo ingestion, which would have indicated that there was no change in the bifidobacteria between these conditions. Despite the characteristics of the cluster analysis shown 
on the dendrogram, each participant's position on the dendrogram remained largely unchanged by placebo or lactulose ingestion. This indicates that the increase in bifidobacteria following lactulose intake cannot be accounted for by particular bifidobacterial species; the numbers of each bifidobacterial species increased to a similar extent. Thus, variations in the composition of the bifidobacteria within individuals do not exceed the differences between individuals during lactulose intake. The combinations of bifidobacterial species and their ratios might be of significance, but further research is needed to explore this. In particular, the B. catenulatum group showed a constantly higher log cell count than the $B$. longum group in cluster 2 . When comparing cluster 2 with cluster 1 , the $B$. catenulatum group did not predominate in the presence of the $B$. adolescentis group, but did predominate in the absence of the $B$. adolescentis group. This indicates that the abundance of the $B$. catenulatum group may be affected by the $B$. adolescentis group.

Furthermore, PCoA based on the intestinal microbiota analysis data showed no significant difference between lactulose ingestion and placebo ingestion. Analysis of the intestinal microbiota revealed that the abundance of the bifidobacteria was significantly higher following lactulose ingestion, whereas those of other intestinal bacterial taxa did not significantly change. These results suggest that lactulose ingestion significantly increases the abundance of HRB, but does not significantly affect particular intestinal microbial taxa. Because lactulose ingestion increases the amount of hydrogen gas in the breath, it is presumed that intestinal bacteria other than bifidobacteria, which do not generate hydrogen, also utilise lactulose for growth. When lactulose is metabolised by the caecal microbiota, the intestinal pH is reduced (Bown et al., 1974), and this is partly due to the acetic acid produced by Bifidobacterium. Because acetic acid has high bacteriostatic activity, it is likely that the growth of intestinal bacteria other than bifidobacteria is suppressed, which causes bifidobacteria to predominate. In other words, the increase in the abundance of bifidobacteria that occurs following lactulose ingestion may not be attributable to the suppression of the growth of other intestinal bacteria by lactulose itself, but may simply be due to greater nutrient availability. If this is the case, the increase in bifidobacterial numbers would not greatly affect the composition of the intestinal microbiota as a whole. It is speculated that only a large amount of bifidobacteria was significantly increased as a result of the gradual decrease of many intestinal bacteria other than bifidobacteria within a range in which no statistically significant difference was obtained.

A limitation of the present study is that we were unable to clarify the roles of individual bifidobacterial species. Studies aimed at better understanding the detailed relationships between bifidobacterial species, and also between the wider intestinal microbiota and bifidobacteria, may clarify the health benefits of bifidobacteria in the future.

The present findings show several patterns in the combinations of bifidobacterial species that are present during lactulose supplementation, which suggests that there are probably distinct effects of bifidobacterial species. Furthermore, we show that lactulose ingestion leads to an increase in HRBs in the intestines, but this greater abundance of bifidobacteria does not affect the intestinal microbiota as a whole.

\section{Supplementary material}

Supplementary material can be found online at https://doi. org/10.3920/BM2020.0100.

Table S1. PCR primers used to detect each bifidobacterial species.

Table S2. Observations in each cluster.

Figure S1. Numbers of bifidobacterial species.

Figure S2. Detection rate and cumulative cell counts of bifidobacterial species in each cluster of the dendrogram.

\section{Acknowledgements}

We greatly appreciate the cooperation of the study participants, who understood the significance of this research, and the staff of Showa Women's University Laboratory of Microbiology for their assistance. We would like to thank Dr Odamaki of Next Generation Science Institute, Morinaga Milk Industry Co., Ltd, for advice regarding this study. This research was made possible with funding from Morinaga Milk Industry Co., Ltd.

\section{Conflict of interest}

There is no conflict of interest to disclose.

\section{References}

Ballongue, J., Schumann, C. and Quignon, P., 1997. Effects of lactulose and lactitol on colonic microflora and enzymatic activity. Scandinavian Journal of Gastroenterology 32, Suppl. 222: 41-44. https://doi.org/10.1080/00365521.1997.11720716

Bouhnik, Y., Attar, A., Joly, F.A., Riottot, M., Dyard, F. and Flourie, B., 2004a. Lactulose ingestion increases faecal bifidobacterial counts: a randomised double-blind study in healthy humans. European Journal of Clinical Nutrition 58: 462-466. https://doi.org/10.1038/ sj.ejcn.1601829 
Bouhnik, Y., Raskine, L., Simoneau, G., Vicaut, E., Neut, C., Flourié, B., Brouns, F. and Bornet, F.R., 2004b. The capacity of nondigestible carbohydrates to stimulate fecal bifidobacteria in healthy humans: a double-blind, randomized, placebo-controlled, parallel-group, dose-response relation study. American Journal of Clinical Nutrition 80: 1658-1664. https://doi.org/10.1093/ajcn/80.6.1658

Bown, R.L., Gibson, J.A., Sladen, G.E., Hicks, B. and Dawson, A.M., 1974. Effects of lactulose and other laxatives on ileal and colonic $\mathrm{pH}$ as measured by a radiotelemetry device. Gut 15: 999-1004. https:// doi.org/10.1136/gut.15.12.999

Callahan, B.J., McMurdie, P.J., Rosen, M.J., Han, A.W., Johnson, A.J.A. and Holmes, S.P., 2016. DADA2: high-resolution sample inference from Illumina amplicon data. Nature Methods 13: 581-583. https:// doi.org/10.1038/nmeth.3869

Choi, K., Hong, T. and Lee, J., 2014. On comparison of SAS codes with GLM and MIXED for the crossover studies with QT interval data. Translational and Clinical Pharmacology 22: 78-82. https:// doi.org/10.12793/tcp.2014.22.2.78

Kato, K., Ishida, S., Tanaka, M., Mitsuyama, E., Xiao, J.Z. and Odamaki, T., 2018. Association between functional lactase variants and a high abundance of Bifidobacterium in the gut of healthy Japanese people. PLoS ONE 13: e0206189. https://doi.org/10.1371/journal. pone.0206189

Kato, K., Odamaki, T., Mitsuyama, E., Sugahara, H., Xiao, J.Z. and Osawa, R., 2017. Age-related changes in the composition of gut Bifidobacterium species. Current Microbiology 74: 987-995. https:// doi.org/10.1007/s00284-017-1272-4

Matsuki, T., Watanabe, K., Fujimoto, J., Kado, Y., Takada, T., Matsumoto, K. and Tanaka, R., 2004. Quantitative PCR with 16S rRNA-gene-targeted species-specific primers for analysis of human intestinal bifidobacteria. Applied and Environmental Microbiology 70: 167-173. https://doi.org/10.1128/AEM.70.1.167-173

Matsuki, T., Watanabe, K., Tanaka, R. and Oyaizu, H., 1998. Rapid identification of human intestinal bifidobacteria by $16 \mathrm{~S}$ rRNAtargeted species- and group-specific primers. FEMS Microbiology Letters 167: 113-121. https://doi.org/10.1111/j.1574-6968.1998. tb13216.x

Matsuki, T., Watanabe, K., Tanaka, R., Fukuda, M. and Oyaizu, H., 1999. Distribution of bifidobacterial species in human intestinal microflora examined with 16S rRNA-gene-targeted species-specific primers. Applied and Environmental Microbiology 65: 4506-4512. https://doi.org/10.1128/AEM.65.10.4506-4512.1999

Mizota, T., Mori, T., Yaeshima, T., Yanagida, T., Iwatsuki, K., Ishibashi, N., Tamura, Y. and Fukuwatari, Y., 2002. Effects of low dosages of lactulose on the intestinal function of healthy adults. Milchwissenschaft 57: 312-315.
Nishijima, S., Suda, W., Oshima, K., Kim, S.W., Hirose, Y., Morita, H. and Hattori, M., 2016. The gut microbiome of healthy Japanese and its microbial and functional uniqueness. DNA Research 23: 125-133. https://doi.org/10.1093/dnares/dsw002

Odamaki, T., Kato, K., Sugahara, H., Hashikura, N., Takahashi, S., Xiao, J.Z., Abe, F. and Osawa, R., 2016. Age-related changes in gut microbiota composition from newborn to centenarian: a crosssectional study. BMC Microbiology 16: 90. https://doi.org/10.1186/ s12866-016-0708-5

Sakai, Y., Seki, N., Hamano, H., Ochi, H., Abe, F., Masuda, K. and Iino, H., 2019a. Prebiotic effect of two grams of lactulose in healthy Japanese women: a randomised, double-blind, placebo-controlled crossover trial. Beneficial Microbes 10: 629-639. https://doi. org/10.3920/BM2018.0174

Sakai, Y., Seki, N., Hamano, H., Ochi, H., Abe, F., Shimizu, F, Masuda, K. and Iino, H., 2019b. A study of the prebiotic effect of lactulose with low dosages on healthy Japanese women. Bioscience of Microbiota, Food and Health 38: 69-72. https://doi.org/10.12938/bmfh.18-013 Sugahara, H., Odamaki, T., Hashikura, N., Abe, F. and Xiao, J.Z., 2015. Differences in folate production by bifidobacteria of different origins. Bioscience of Microbiota, Food and Health 34: 87-93. https://doi. org/10.12938/bmfh.2015-003

Tomoda, T., Yasuo, N. and Takashi, K., 1991. Effect of yogurt and yogurt supplemented with Bifidobacterium and/or lactulose in healthy persons: a comparative study. Bifidobacteria and Microflora 10: 123-130. https://doi.org/10.12938/bifidus1982.10.2_123

Tuohy, K.M., Ziemer, C.J., Klinder, A., Knöbel, Y., Pool-Zobel, B.L. and Gibson, G.R., 2002. A human volunteer study to determine the prebiotic effects of lactulose powder on human colonic microbiota. Microbial Ecology in Health and Disease 14: 165-173. https://doi. org/10.1080/089106002320644357

Vanhoutte, T., De Preter, V., De Brandt, E., Verbeke, K., Swings, J. and Huys, G., 2006. Molecular monitoring of the fecal microbiota of healthy human subjects during administration of lactulose and Saccharomyces boulardii. Applied and Environmental Microbiology 72: 5990-5997. https://doi.org/10.1128/AEM.00233-06

Ventura, M., Reniero, R. and Zink, R., 2001. Specific identification and targeted characterization of Bifidobacterium lactis from different environmental isolates by a combined multiplex-PCR approach. Applied and Environmental Microbiology 67: 2760-2765. https:// doi.org/10.1128/AEM.67.6.2760-2765.2001

Wong, C.B., Sugahara, H., Odamaki, T. and Xiao, J.Z., 2018. Different physiological properties of human-residential and non-humanresidential bifidobacteria in human health. Beneficial Microbes 9: 111-122. https://doi.org/10.3920/BM2017.0031

Yaeshima, T., 1996. Benefits of bifidobacteria to human health: oligosaccharides and probiotic bacteria. Bulletin of the International Dairy Federation 313: 36-42. 
